\title{
APPLICATION OF QUALITATIVE REASONING IN ENGINEERING
}

\author{
LUIS M. BOZZO \\ Universidad de Girona, Escuela Politécnica Superior, \\ Girona, Spain
}

ALEX BARBAT

Universidad Politécnica de Catalunya, ETS Ingenieros de Caminos, Canales y Puertos, Barcelona, Spain

\section{LLUIS TORRES}

Universidad de Girona, Escuela Politécnica Superior, Girona, Spain

\begin{abstract}
Qualitative reasoning is an alternative problem-solving technique useful for the conceptual design of structures. Qualitative reasoning represents the relationships between parameters in a model, and a search computation assigns values represented by intervals and relevant points in the behavior. The traditional difference between analysis and design or input and output parameters in a procedural computation is not existent in qualitative reasoning, since all the parameters in a model are equally represented. Qualitative reasoning derives values for parameters even with incomplete and imprecise knowledge about the model. This work presents a qualitative structural analysis framework, suitable for the evaluation of conceptual designs as well as for tutoring systems. The framework has been implemented in a computer program called Agrippa using the computer language Prolog. Based on a representation of fundamental principles of equilibrium, compatibility, and force-deformationand an incomplete knowledge of geometry and topology, Agrippa derives the signs and relative magnitude of forces and displacements for three-dimensionalmodels of structures.
\end{abstract}

Conceptual design is one of the most important stages of the design process because it determines the overall behavior of a structure. Many sound structural designs have been constructed in the past based purely on good conceptual understanding of basic fundamental laws, without the precise numerical models available at present. Numeric solutions to governing equations provide detailed information about the structural and nonstructural demands. However, the detailed information required for a quantitative analysis may not be available, or the influence of factors such as material characteristics, soil conditions, and influence of nonstructural components may not be fully investigated. Therefore emphasis on conceptual

Received April 1996; revised November 1996.

Part of this research was supported by project "Técnicas cualitativas, numéricas y simbólicas en diseño asistido por ordenador" by the Spanish government.

Address correspondence to Luis M. Bozzo, Universidad de Girona, Escuela Politécnica Superior, Av. Lluis Santalo s/n (Mód. P2), 17003 Girona, Spain. 
designs rather than on numeric calculations is one way to achieve good solutions during the design task.

In general, qualitative reasoning is a field of research that explains how the model of a device functions by representing the fundamental or first principles for the domain (De Kleer \& Brown, 1984). Qualitative reasoning derives behavior from the description of a model and does not rely, necessarily, on heuristic knowledge. Compared to the shallow level of knowledge represented in most heuristic rules, qualitative reasoning frameworks are called second-generation expert systems because of the representation of fundamental principles.

Quantitative or numeric models represent fundamental principles of the domain by using algebraic or differential equations, but there are major differences between quantitative and qualitative or nonnumeric models. For quantitative models the parameters are in a large, usually infinite, range of values such as the set of real numbers. Given complete information about the input parameters, a procedural computation results in a unique solution, which is valid only for the selected parameters. Even a small variation in the model requires a new analysis or, at the very least, an evaluation of the sensitivity coefficients. For qualitative models, in contrast, parameters are represented by a small set of values such as positive, zero, and negative. Given possibly incomplete information about the qualitative values of the parameters, a search results in a usually nonunique set of solutions. Qualitative models represent the relationships between parameters, and the classical distinction
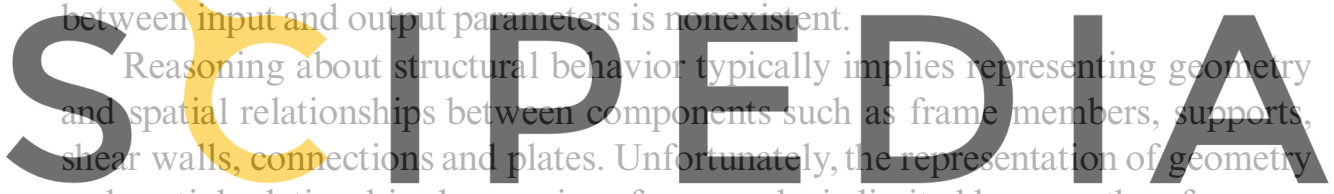

and spatial relationships by previous frameworks is limited because they focus on

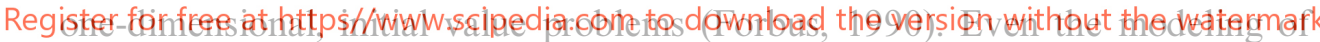

simple one-dimensional structural engineering problems such as strings in parallel or in series is cumbersome and inefficient to handle using previous frameworks (Bozzo \& Fenves, 1992). The necessity for a qualitative reasoning framework more suitable to describe and represent spatial quantities has been previously stated by at least Forbus (1990) and Cohn (1987). These observations motivate the development of the reasoning framework presented next.

\section{REASONING FRAMEWORK FOR QUALITATIVE STRUCTURAL ANALYSIS}

Given a model of a structure and a set of parameters representing its behaviorsuch as rotations and bending moments - the qualitative structural analysis problem may be stated as the search for the unknownqualitative values and their relationships that satisfy the laws of equilibrium, compatibility, and force-deformation. The 
qualitative values are intervals or relevant points such as \{negative, zero, positive useful to understand the behavior of a structure. The absolute magnitude of the parameters is also ordered with respect to other magnitudes by relations such as \{greater, equal, smaller\}. Consequently, in qualitative structural analysis a parameter is represented by its sign and by its relationship in magnitude with other parameters in the model. The indicated values, along with their relationships, provide the important characteristics of the forces and displacements to reason about structural behavior.

Consider the continuous beam illustrated in Figure $1 a$. Following the aforementioned definition, the qualitative structural analysis problem consists in searching for qualitative values and parameter relationships for the connection rotations, bending moments, and shear forces that satisfy the fundamental laws. Figures $1 b$ and $1 c$ illustrate a valid qualitative solution establishing the qualitative values and their relationships for the bending moment diagram and deflected shape, respectively. The qualitative solution for this simple problem indicates that the connection bending moments and rotations diminish at increasing distances from the load, as illustrated in the figure.

\section{Component States}

A structure is formed by components and their connections. A component may be an individualcomponent, such as a column, beam, or shear wall, or an aggregation

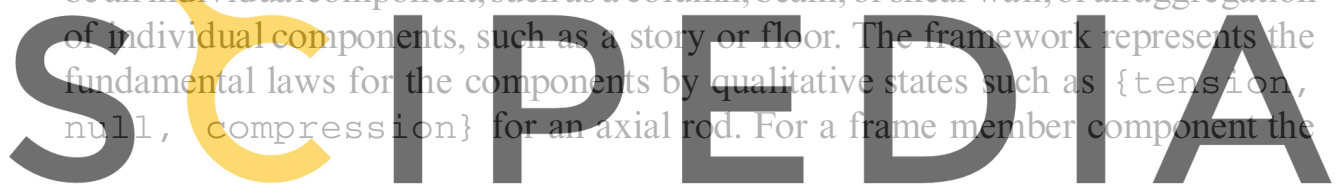

Register for freqa.

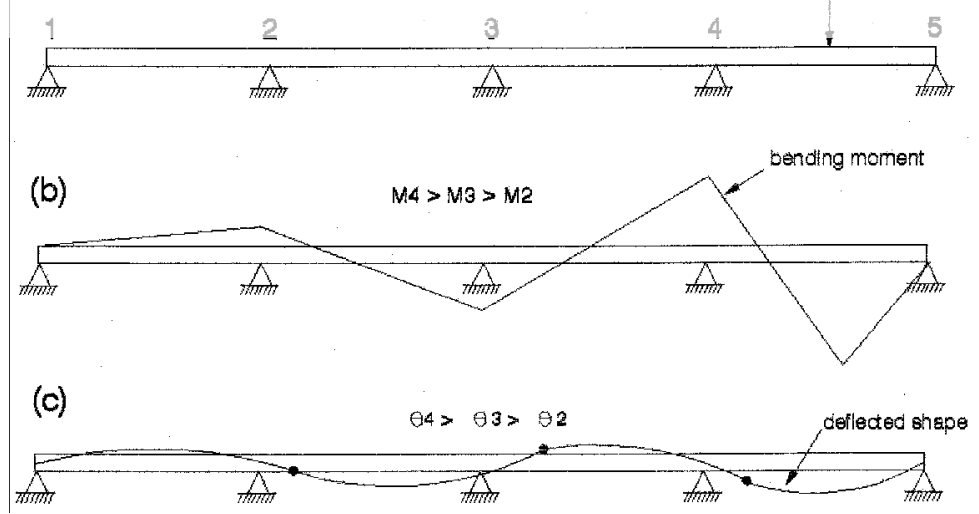

Figure 1. (a) Continuous beam with concentrated load. The material characteristics and section are constant through the length. (b) Qualitative solution for bending moments. (c) Qualitative deflected shape. 
equilibrium laws establish a relation between the forces and moments at the two component connections. To illustrate the relations, consider the equilibrium laws at a plane for the frame member:

\begin{tabular}{ll} 
Axial forces & Bending moments and shear forces \\
\hline$F_{x}^{1}+F_{x}^{2}=0$ & $F_{z}^{1}+F_{z}^{2}=0$ \\
& $M_{y}^{1}+F_{z}^{1} L+M_{y}^{2}=0$
\end{tabular}

where $F_{x}^{i}, F_{z}^{i}, M_{y}^{i}$, and $L$ are axial forces, shear forces, bending moments, and component length, respectively. The superscripts 1 and 2 represent the initial and final connections for the frame component. The notation and local axis definitions are illustrated in Figure 2.

Using the aforementioned qualitative values nnegative, zero, positive\}, a frame member component has 17 states with respect to the equilibrium between bending moments and shear forces (Bozzo \& Fenves, 1992). Each of these states prescribes the qualitative values of the forces and moments and the parameter relations between the two connections. Besides these 17 states, there are 3 states for axial forces (tension, compression, and null force), which are independent of the bending states.

The conjugate beam analogy recognizes the similarity between the equilibrium and compatibility laws (Oden, 1967). The analogy has been extensively used to teach

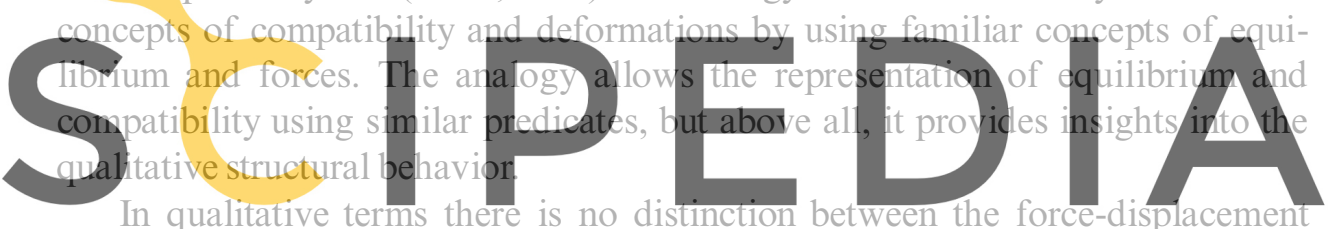

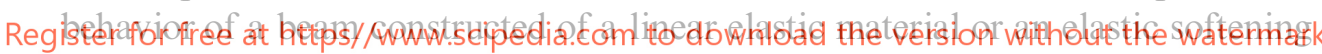
material. This is illustrated in Figure 3 by using the conjugate beam analogy. For a linear material, if the moment $M_{1}$ is greater than the moment $M_{2}$, then the conjugate load $F_{1}$ is also greater than the conjugate load $F_{2}$, and an elastic softening material accentuates this relationship. A similar conclusion is derived for the relations between displacements because softening translates the center of gravity of the conjugate loads closer to the ends of the member. By taking into account rotations,

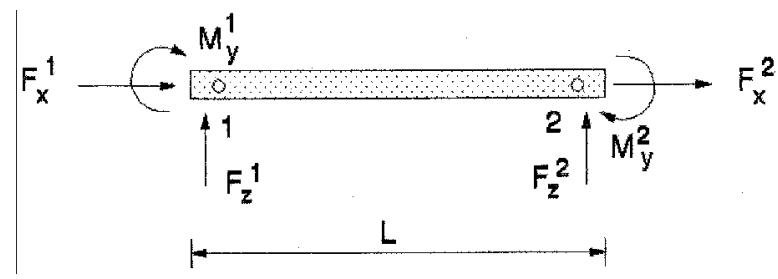

Figure 2. Sign convention and local coordinate axis for a frame component. 


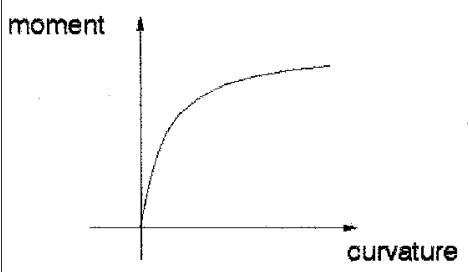

section force-deformation

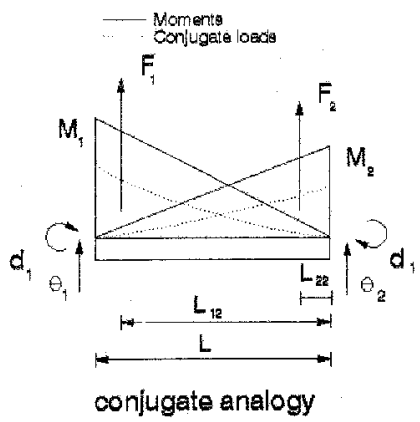

Figure 3. Conjugate beam analogy for the compatibility and force-displacement laws.

there are 25 bending related states for a frame member component, which are illustrated in Figure 4.

\section{Connection Processes}

Equilibriumand compatibility at a connection are represented by processes that change the component states attached to the connection. The representation avoids the necessity to define a particular component for the large number of possible connections such as one made up by two components or another made up by four components.

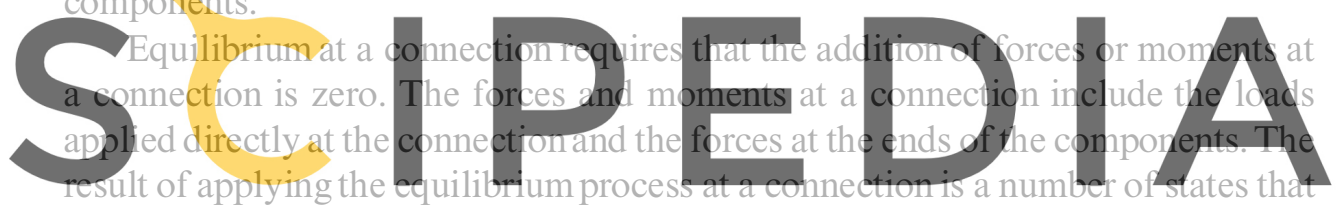

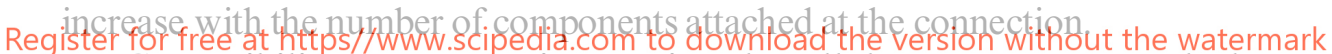

Compatibility at a connection requires that all the components attached to a

connection have the same qualitative rotations and displacements. This is directly achieved in the reasoning framework defining the displacements of the connections as the parameters of the model, as is common practice in numeric structural analysis computer programs. In this way, the displacements and rotations for any component attached to a connection are those of the connection.

\section{Inference Scheme}

A fundamental aspect of the reasoning framework is the inference scheme, which defines how to combine the parameter values resulting from the component states and the connection processes. The inference scheme for the reasoning framework consists of two steps: (1) the elaboration step that augments an initial description by adding qualitative values that follow from the initial description and orders the components for efficiency in the inference and (2) the solution propagation step, 


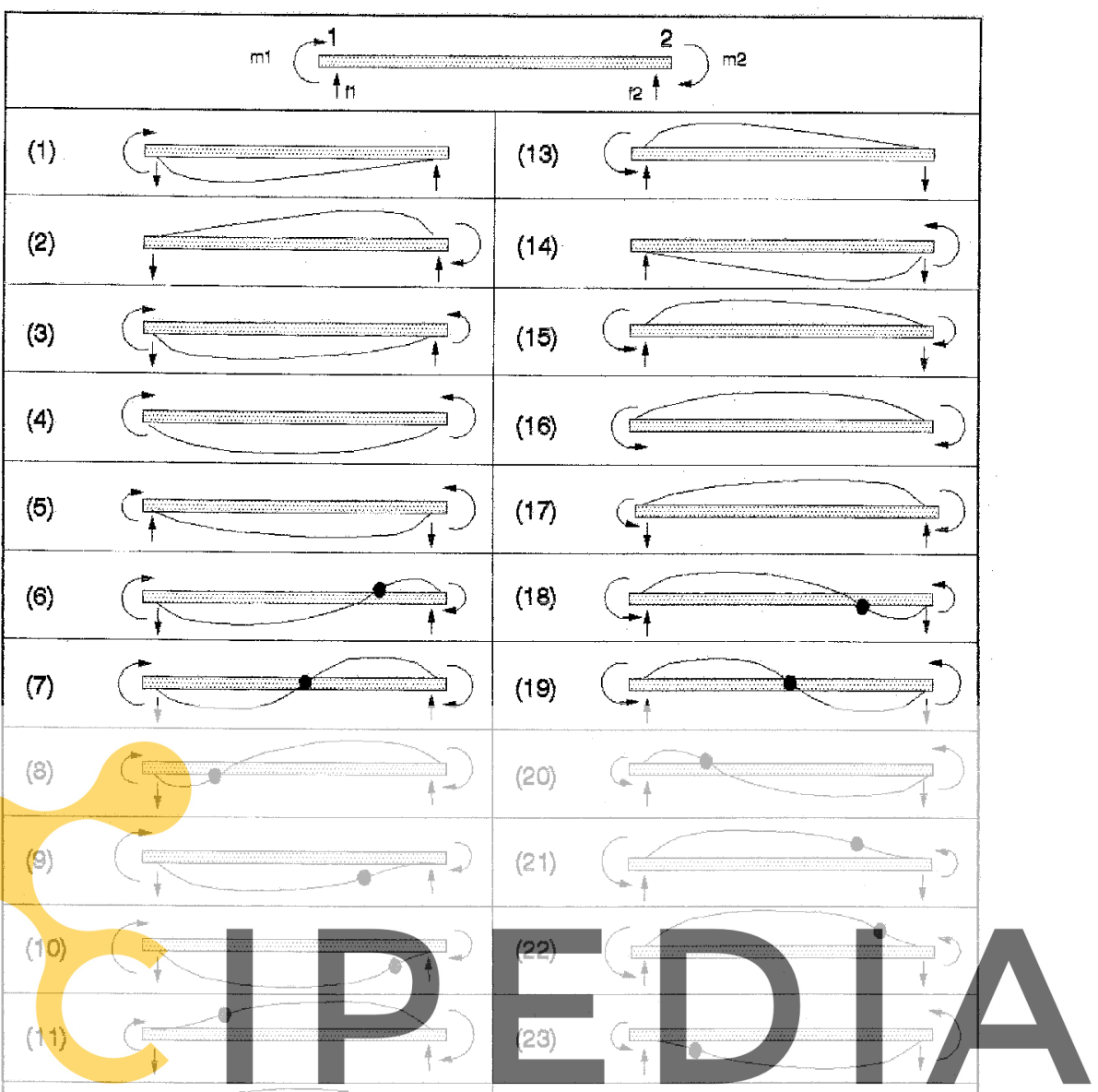

Register for ffee at htips//www.scipedia.com to download the version without the watermark

(25)

Figure 4. Qualitative bending states for a frame member component.

which completes the initial description deriving values for unknown parameters in the model. The solution propagation employs a backward-forward inference technique.

The elaboration step is performed once at the beginning of the inference process. This step enhances an initial model description given by the user and infers as much information as possible from it. The elaboration step also orders the components in increasing number of states in order to improve the efficiency in the inference. A motivation behind the elaboration step is that an intelligent problem solver should 
derive as much obvious information as possible from the initial problem description. Therefore the elaboration incorporates default values for the components in the model. For example, the out-of-plane forces or displacements for a planar frame are zero, and consequently, these zero values are added into the solution. Other default values are the zero displacements and rotations for a fixed support. The second step during the elaboration is the initial forward propagation. This step considers each one of the components in the model and executes a forward inference procedure. The forward propagation infers values consistent with the fundamental laws, which are derived without ambiguity. The forward propagation is a fundamental part of the backward-forward solution propagation, and therefore it is explained in more detail next.

The elaboration step has two important features besides the obvious advantages in execution efficiency: (1) it may detect structural instabilities because of a lack of an equilibrium solution, early in the reasoning process, and (2) the reasoning process for many statically determinate structures is reduced to the elaboration step.

The solution propagation is invoked if the elaboration step does not provide a full description of the parameter values, which is typically the case for a statically indeterminate structure. The solution propagation uses backward and forward chaining. The solution propagation starts by assuming qualitative values for a component in the model in a backward chaining propagation. Using this new information, the forward chaining propagation derives values from the new information, added by the backward chaining propagation.

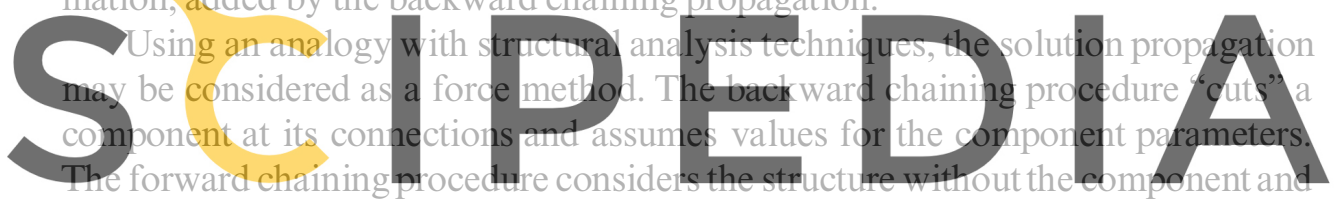

applies the assumed forces, and displacements at the component connections as, prescribed forces and displacements. If the cut transforms the structure into a

statically determinate structure, the forward chaining procedure may infer values to all the parameters in the structure. If the forward chaining procedure does not derive values for all the parameters in the model, the backward chaining procedure makes another cut. The backward chaining propagation proceeds until it reaches a complete description of the parameters in the model.

The backward or goal-driven procedure starts with a list of component whose parameter values are (1) given by the user, or (2) derived by the default values or by the initial forward propagation during the elaboration step, or (3) ambiguous. It is called a backward or goal-driven procedure because it derives values by using a backward chaining inference. The backward chaining procedure assigns a valid qualitative state for a component and for the parameters defined at the connections between components that satisfy the connection processes.

A component has a number of states that satisfy the laws of the domain, as the 25 states for the bending related states for a frame member illustrated in Figure 4. 
The first objective of the backward propagation procedure is to select the component states that are consistent with the known values for the component parameters. For example, consider a frame member and assume some parameter values are known, as Figure 5 illustrates. Using the known values, the component states are reduced from the original 25 to only 2 : one for a positive bending moment and one for a negative bending moment. The state corresponding to zero bending moment is not considered in this example. Using the available information to reduce the number of component states considerably increases the efficiency of the inference process because the known information reduces the search space.

Table 1 presents the initially known values and the two component states from Figure 5, in a representation that is useful to explain subsequent examples. At each frame member connection, one six-tuple represents forces and moments according to the convention $\left(F_{x}, F_{y}, F_{z}, M_{x}, M_{y}, M_{z}\right)$, and another six-tuple represents displacements and rotation according to the convention $\left(\theta_{x}, \theta_{y}, \theta_{z}, d_{x}, d_{y}, d_{z}\right)$.

The parameter relations between forces are not included in Table 1 because forces along a frame member are considered as being constant and represented by a unique parameter. Parameter relations between displacements, which all have zero values, are also not included in the table.

The backward chaining procedure selects the first valid component state or state 1 in Table 1. If the connection processes or the qualitative calculus detects an inconsistency, then the backward chaining procedure disregards the state 1 and selects the second valid component state, or state 2 in Table 1.

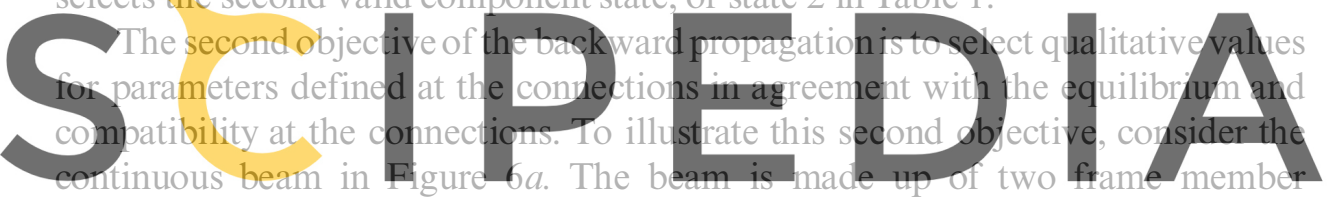

components, each with exactly the same states preyiously presented. The backward, Register for free at https//www.scipedia.com to downfoad the version without the watermark propagation for the components assumes the first valid state, or state 1 in Table 1 , for the left component. Using this information, the backward propagation for the

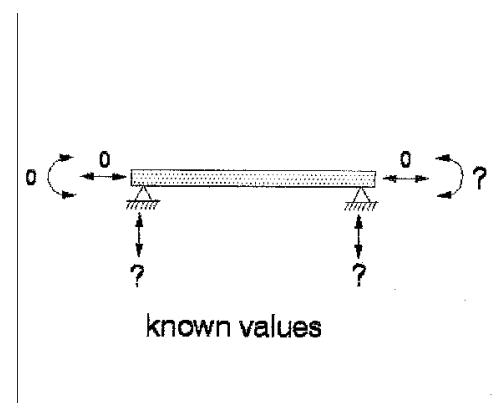

state 1

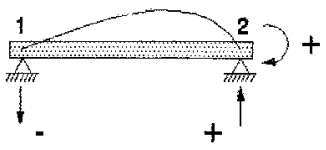

Figure 5. Known parameter values and derived component states for a frame member. 
Table 1. Qualitative states for a frame member

\begin{tabular}{|c|c|c|c|c|c|c|}
\hline & \multicolumn{2}{|c|}{ Force } & \multicolumn{2}{|c|}{ Displacement } & \multicolumn{2}{|c|}{ Relation } \\
\hline & Node 1 & Node 2 & Node 1 & Node 2 & Displacements & Forces \\
\hline Initial values & $(0,0, ?, 0,0,0)$ & $(0,0, ?, 0, ?, 0)$ & $(0, ?, 0,0,0,0)$ & $(0, ?, 0,0,0,0)$ & & \\
\hline State 1 & $(0,0,-, 0,0,0)$ & $(0,0,+, 0,+, 0)$ & $(0,-, 0,0,0,0)$ & $(0,+, 0,0,0,0)$ & $\left|\theta_{y}^{2}\right|>\left|\theta_{y}^{1}\right|$ & $\left|M_{y}^{2}\right|>\left|M_{y}^{1}\right|$ \\
\hline State 2 & $(0,0,+, 0,0,0)$ & $(0,0,-, 0,-, 0)$ & $(0,+, 0,0,0,0)$ & $(0,-, 0,0,0,0)$ & $\left|\theta_{y}^{2}\right|<\left|\theta_{y}^{1}\right|$ & $\left|M_{y}^{2}\right|<\left|M_{y}^{1}\right|$ \\
\hline
\end{tabular}

connection laws derives three possible values for the forces and displacements at the middle connection, as illustrated in Figure $6 b$ and Table 2.

The forward or data-driven propagation procedure is executed after the backward chaining inference procedure. It is named data driven because it uses the newly added qualitative values from the backward procedure to infer values that are not ambiguous. As for backward chaining, it derives values according to the fundamental laws. The differences with backward chaining are that forward chaining infers values that are not ambiguous, and the goals are executed following paths along the structure.

A structure is represented as a graph formed by components and connections. Each time the connection processes add values for forces or displacements, a procedure, similar to depth-first search, traverses the paths from the connection. The difference with "depth first" is that a node (a component or a connection in the
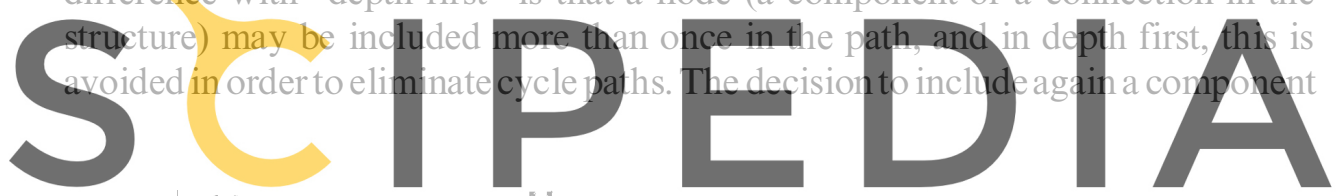

(a)

Register for free at https//4www.şcipedia ${ }_{2}$ com $_{1}$ to dogwnload the version without the watermark

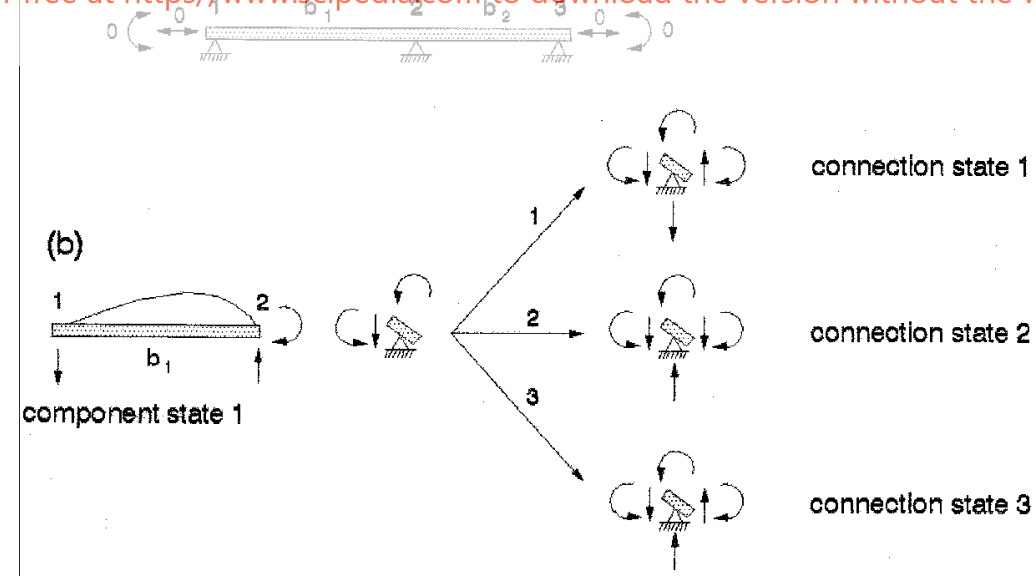

Figure 6. (a) Continuous beam. (b) Values inferred by the backward propagation of compatibility and equilibrium at the middle connection. 
Table 2. Qualitative values resulting from state one for component $b 1$ in Figure 6

$b 1$

\begin{tabular}{|c|c|c|c|c|c|c|c|c|}
\hline \multirow[b]{2}{*}{ Connection } & \multicolumn{2}{|c|}{ Force } & \multicolumn{2}{|c|}{ Displacement } & \multicolumn{2}{|c|}{ Force } & \multicolumn{2}{|c|}{ Displacement } \\
\hline & Node 1 & Node 2 & Node 1 & Node 2 & Node 2 & Node 2 & Node 3 & Node 3 \\
\hline 1 & $(0,0,-, 0,0,0)$ & $(0,0,+, 0,+, 0)$ & $(0,-, 0,0,0,0)$ & $(0,+, 0,0,0,0)$ & $(0,0,-, 0,-, 0)$ & $(0,0, ?, 0, ?, 0)$ & $(0,+, 0,0,0,0)$ & $(0, ?, 0,0,0,0)$ \\
\hline 2 & $(0,0,-, 0,0,0)$ & $(0,0,+, 0,+, 0)$ & $(0,-, 0,0,0,0)$ & $(0,+, 0,0,0,0)$ & $(0,0,+, 0,-, 0)$ & $(0,0, ?, 0, ?, 0)$ & $(0,+, 0,0,0,0)$ & $(0, ?, 0,0,0,0)$ \\
\hline 3 & $(0,0,-, 0,0,0)$ & $(0,0,+, 0,+, 0)$ & $(0,-, 0,0,0,0)$ & $(0,+, 0,0,0,0)$ & $(0,0,-, 0,-, 0)$ & $(0,0, ?, 0, ?, 0)$ & $(0,+, 0,0,0,0)$ & $(0, ?, 0,0,0,0)$ \\
\hline
\end{tabular}

or a connection in the path depends on the fact that the component or connection adds, again, new qualitative values or not. Consequently, the procedure always finishes.

The result of each depth-first propagation is (1) the path leads to ambiguity and therefore is not continued; (2) the path leads to the resolution of qualitative values that are consistent with previous information, and those values are accepted into the system description; and (3) the path leads to the resolution of qualitative values that are not consistent with previous information, and the assumed state of the component is rejected. This outcome implies leaving the forward propagation procedure and returning to the backward chaining procedure.

As an example, consider the continuous beam and the three solutions generated by the backward propagation illustrated in Figure 6 and Table 2. The forward
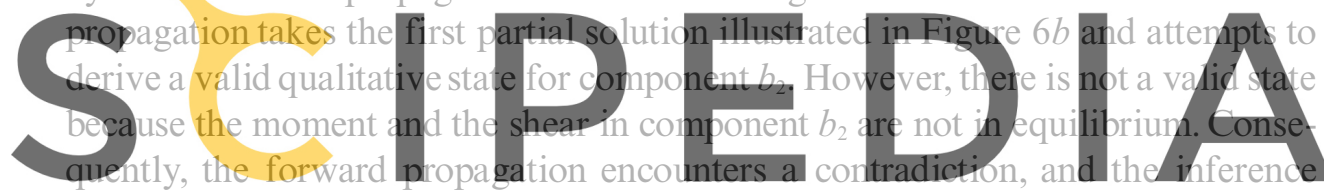

scheme returns to the backward chaining propagation and takes the second partial Register for free at https fw ww scipediacom to download the versjon. without the waternark

state for component $b_{2}$, but again there is not a valid state because connection 2 has a positive rotation but the left moment for component $b_{2}$ causes a negative rotation at connection 2. Similarly, the solution number three from the backward chaining procedure is not correct because there is no equilibrium between the shear and the moment for component $b_{2}$. The backward propagation is invoked, and the second state for component $b_{1}$ is assumed. This state corresponds to the real solution, and consequently, the inference scheme does not detect a contradiction.

Table 3 presents an estimation of various inference strategies applied to the simple continuous beam illustrated in Figure $1 a$. There are 16 parameters -5 connection rotations, 6 bending moments, and 5 shear forces - and consequently, a pure parameter combination search results in $3^{16}$ cases for the qualitative values \{negative, zero, positive\}. As indicated previously, an unloaded frame member component has 25 component states. Taking into account that a loaded component has about 41 states, a pure state combination inference strategy results 
Table 3. Estimation of the number of combinations to find the qualitative solutions for various inference strategies applied to the beam in Figure $1 a$

\begin{tabular}{llll}
\hline \multirow{2}{*}{$\begin{array}{l}\text { Pure parameter } \\
\text { combination }\end{array}$} & $\begin{array}{l}\text { No elaboration, pure } \\
\text { states combination }\end{array}$ & \multicolumn{2}{c}{ Elaboration and backward-forward chaining } \\
\cline { 3 - 4 } & $41 \times 25 \times 25 \times 25$ & $41 \times 12 \times 12 \times 1$ & Ordered \\
\hline $3^{16}$ & 40 ordered & 2 \\
\hline
\end{tabular}

in $41 \times 25 \times 25 \times 25$ cases. Even though this number is much smaller than $3^{16}$, it is still a very large number, especially taking into account the simplicity of the problem and that there is just one correct qualitative solution illustrated in Figures $1 b$ and $c$.

A significant reduction in the number of combinations is achieved if the proposed inference scheme is employed. Suppose the inference scheme follows the path from component $b_{45}$ to component $b_{1-2}$, i.e., the elaboration step does not order the components in increasing number of states. As in a pure state combination inference scheme, component $b_{4.5}$ has about 41 states-rigorously speaking, less than 41 because of the zero-moment condition at connection 5 . Once the inference scheme assumes a state for component $b_{4-5}$, the states for components $b_{2-3}$ and $b_{3-4}$ are reduced from 25 to about 12 . Equilibrium and compatibility and the initially known qualitative values also reduce the states for component $b_{1-2}$ from 25 to only 1. Consequently, an inference strategy that starts with component $b_{45}$ and ends with component $b_{1-2}$ has about $41 \times 12 \times 12 \times 1$ combinations. Finally, if the elaboration step orders the components in increasing number of states, the inference scheme would start with component $b_{1-2}$. This inference scheme reduces the number of combinations drastically, since the total number of combinations is only 2 . The reduction is possible, since there is just one valid state for component $b_{2-3}$, once the rotation and bending moment for component $b_{1-2}$ are known.

\section{Verification of Solution Consistency}

A very important aspect of the reasoning framework is how to verify that a given qualitative solution fully satisfies the domain laws. Individual components and their connections directly satisfy the laws; however, free body diagrams of an aggregation of components may not satisfy them. To illustrate the problem, consider a model where the lengths and material characteristics are known numeric values. The laws of compatibility, equilibrium, and force-deformation are transformed into linear equations: a numerical procedure may easily solve this problem, obtaining the precise numeric values for the displacements and forces in the model. A qualitative solution represented by a set of algebraic relationships, such as $F 1=$ positive and $F 1>F 2$, is valid or "consistent" if there is no contradiction between the aforementioned algebraic relationships and the linear equations representing the fundamental laws. 
Obviously, in a model where the lengths and material characteristics are known numeric values, there is no point in performing qualitative structural analysis. However, the illustration is useful to establish the general verification of consistency problem: demonstrate there is no contradiction between a given set of nonlinear algebraic relationships for the fundamental laws and a set of linear algebraic relationships for the qualitative solution. The proposed reasoning framework uses four techniques to verify the consistency of a set of qualitative values and parameter relations. The techniques are basic qualitative calculus operations, transitivity rules, constant elimination, and consistency checking (Bozzo \& Fenves, 1992). Rigorously speaking, the first two techniques are particular cases of the constant elimination technique. However, because of efficiency considerations - which are not discussed in detail in this work - it is convenient to present them separately.

The first technique, basic qualitative calculus operations, adds or subtracts qualitative values in analogous operations as for real numbers. The technique takes into account the parameter relations between the qualitative values in order to reduce ambiguity. For example, the addition of a positive and a negative value is ambiguous unless the parameter relation between them is considered.

Transitivity rules is a technique based on a set of three axioms useful to propagate parameter relations. This technique derives relations such as if $A>B$, $B>C$, then $A>C$. The axioms are

$$
\begin{array}{ll}
A>B & B>C \Rightarrow A>C \\
A=B & B>C \Rightarrow A>C \\
A=B & B=C \Rightarrow A=C
\end{array}
$$

Linear equations are those expressed as the addition of parameters without multiplications or divisions between them. Constant elimination is a simple algebraic simplifier that derives new equations based on existing ones (Bredeweg et al., 1990). In the reasoning framework, constant elimination is applied to linear equations such as the equilibrium equations at a connection. For example, the constant elimination procedure derives the relation $F 1+F 2=F 3+F 4$ based on the relations such as $F 1+F 2=P$ and $P=F 3+F 4$. Constant elimination is defined by the following set of axioms:

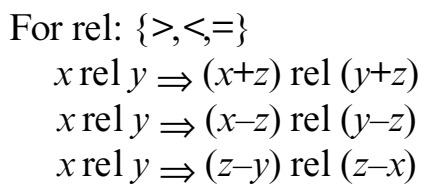

Nonlinear laws are those expressed as the multiplication or division between parameters. For nonlinear laws the reasoning framework uses a technique called 
consistency checking. This technique is defined by the following set of axioms similar to the axioms for constant elimination:

$$
\begin{aligned}
& \text { For rel: }\{>,<,=\} \\
& \begin{aligned}
z>0 \text { and } x \text { rel } y \Rightarrow\left(x^{*} z\right) \operatorname{rel}\left(y^{*} z\right) \\
z<0 \text { and } x \text { rel } y \Rightarrow\left(y^{*} z\right) \operatorname{rel}\left(x^{*} z\right) \\
z>0 \text { and } x \text { rel } y \Rightarrow(x / z) \operatorname{rel}(y / z) \\
z<0 \text { and } x \text { rel } y \Rightarrow(y / z) \operatorname{rel}(x / z)
\end{aligned}
\end{aligned}
$$

The difference between constant elimination and consistency checking is how the axioms are applied. Every time a component or a connection is added into the inference scheme, the constant elimination verifies the equilibrium. Consequently, constant elimination is used to verify equilibrium for any free body diagram of the structure. In contrast, consistency checking is activated every time a component is added into the inference, and it verifies compatibility. Consequently, consistency checking verifies compatibility for any path formed by a set of components attached to each other.

The continuous beam in Figure $7 a$ is useful to describe the aforementioned techniques. The solution illustrated in Figure $7 b$ includes the parameter relation

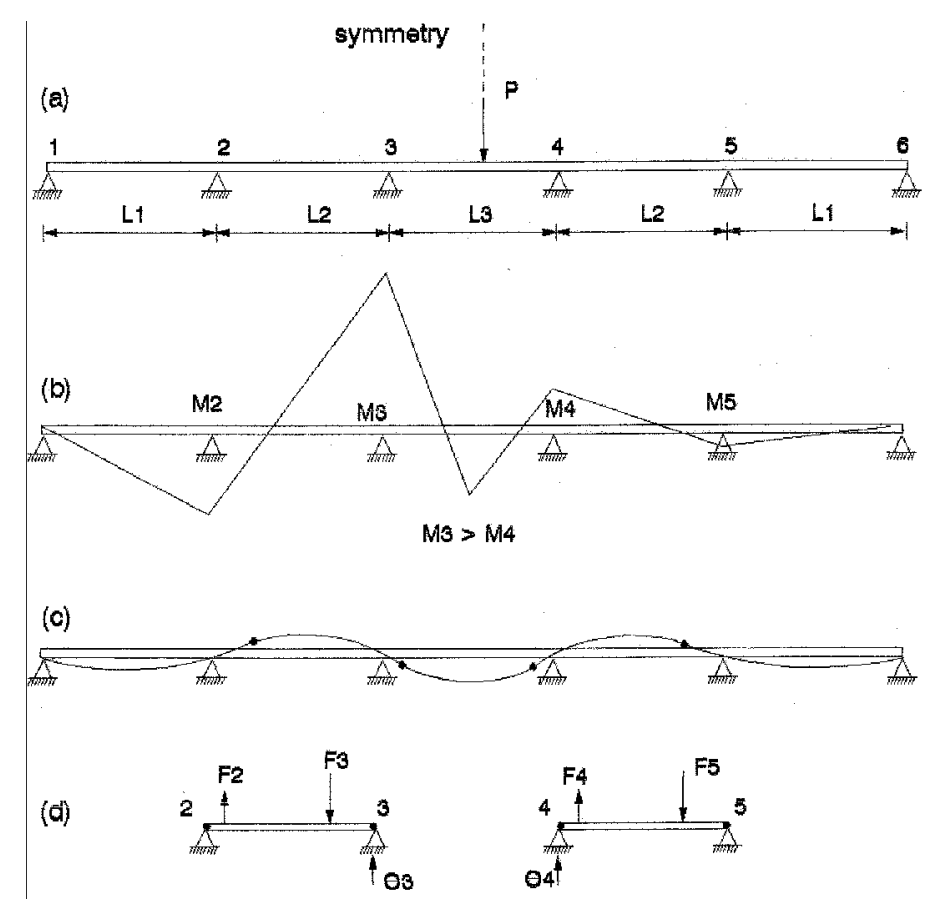

Figure 7. Example of an inconsistent solution detected by the reasoning framework. 
$\left(M_{3}>M_{4}\right)$, which clearly is inconsistent because of symmetry. The goal of the verification procedure is to prove the inconsistency. For tutoring systems an additional goal of the verification may be to explain why it is inconsistent.

Consider initially the loaded component $b_{3-4}$. Taking into account the valid states for the component, it is possible to conclude that a necessary parameter relation is

$$
\left|\theta_{3}\right|<\left|\theta_{4}\right|
$$

Taking into account the component states for the element $b_{2-3}$ illustrated in Figure 4 , it is clear that the unique valid state is the twelfth; the eleventh is not considered, since in general, a zero value is not included in the search. This state adds the following parameter relations:

$$
\left|M_{2}\right|<\left|M_{3}\right| \quad\left|\theta_{2}\right|<\left|\theta_{3}\right|
$$

Similarly, the unique valid state for component $b_{45}$ is the 22 , which adds the parameter relations:

$$
\left|M_{5}\right|<\left|M_{4}\right| \quad\left|\theta_{5}\right|<\left|\theta_{4}\right|
$$

Using the transitivity rules, it is possible to add the following new parameter relations:

$$
\left|M_{3}\right|>\left|M_{5}\right| \quad\left|\theta_{4}\right|>\left|\theta_{2}\right|
$$

Similarly, considering the valid states for components $b_{1-2}$ and $b_{5-6}$ and using transitivity rules, it is easy to add the parameter relations

$$
\left|\theta_{2}\right|>\left|\theta_{1}\right| \quad\left|\theta_{5}\right|>\left|\theta_{6}\right| \quad\left|\theta_{4}\right|>\left|\theta_{1}\right| \quad\left|\theta_{4}\right|>\left|\theta_{6}\right|
$$

The compatibility conditions for components $b_{2-3}$ and $b_{45}$ add the following relationships:

$$
\begin{aligned}
& \frac{2}{3}\left|F_{3}\right| L_{2}=\left|\theta_{3}\right| L_{2}+\frac{1}{3}\left|F_{2}\right| L_{2} \\
& \frac{2}{3}\left|F_{4}\right| L_{2}=\left|\theta_{4}\right| L_{2}+\frac{1}{3}\left|F_{5}\right| L_{2}
\end{aligned}
$$

where $F_{i}=M_{i} L 2 E I$ are the conjugate loads for a linear elastic material as defined in Figure $7 d$. Applying the constant elimination axioms between the latest two expressions and taking into account that 


$$
\left|F_{3}\right|>\left|F_{4}\right| \quad\left|\theta_{3}\right|<\left|\theta_{4}\right|
$$

it is possible to prove without ambiguity that $\left|F_{2}\right|>\left|F_{5}\right|$ and therefore $\left|M_{2}\right|>\left|M_{5}\right|$. Similarly, it is possible to probe that $\left|\theta_{2}\right|<\left|\theta_{s}\right|$. Finally, it is relatively easy to prove the inconsistency between the nonlinear compatibility laws for components $b_{1-2}$ and $b_{5-6}$ and the parameter relations $\left|M_{2}\right|>\left|M_{5}\right|$ and $\left|\theta_{2}\right|<\left|\theta_{5}\right|$.

\section{IMPLEMENTATION}

The reasoning framework for qualitative structural analysis was implemented in the computer program Agrippa using the symbolic language PROLOG. Data structures such as N_PLUS_K trees and AVL trees are extensively used in the implementation(O'Keefe, 1990). N_PLUS_K trees are efficient data structures for storing information whose values do not change during the program execution. If the values change during execution, $A V L$ trees are more efficient data structures.

There are two main features that contribute to the efficiency of Agrippa. The first is the extensive use of indices. In structural engineering a model is made up of several components of the same class with different locations in space. An index is associated with each component, and the index is used to store and retrieve component information from the N_PLUS_K trees.

The second feature that contributes to the efficiency of Agrippa is the inference scheme. The forward propagation of known values based on the topology of the model considerably improves the reasoning process. Inconsistent solutions are detected early in the inference, reducing considerably the backward chaining.

However, Agrippa is a preliminary implementation of the reasoning framework, and computer efficiency was not considered an issue in this research. Presently, there is a project to implement the framework for a tutoring system in structural analysis, which focuses on details to reduce execution times.

\section{APPLICATIONS}

To illustrate the kind of information derived by the program based on an incomplete description of a structure, consider the two-story frame shown in Figure $8 a$. The component's length and section behavior characteristics are unknown except for two facts: the material corresponds to a softening elastic one, and the geometry constraints imply the beams and second-story columns have the same length. To improve the inference efficiency, symmetry is taken into account to model the structure.

An evaluation of the proposed design by Agrippa takes a few seconds and indicates that regardless of the particular value for the lengths or material characteristics, there are three load transfer characteristics, as shown in Figure $8 b$. The 

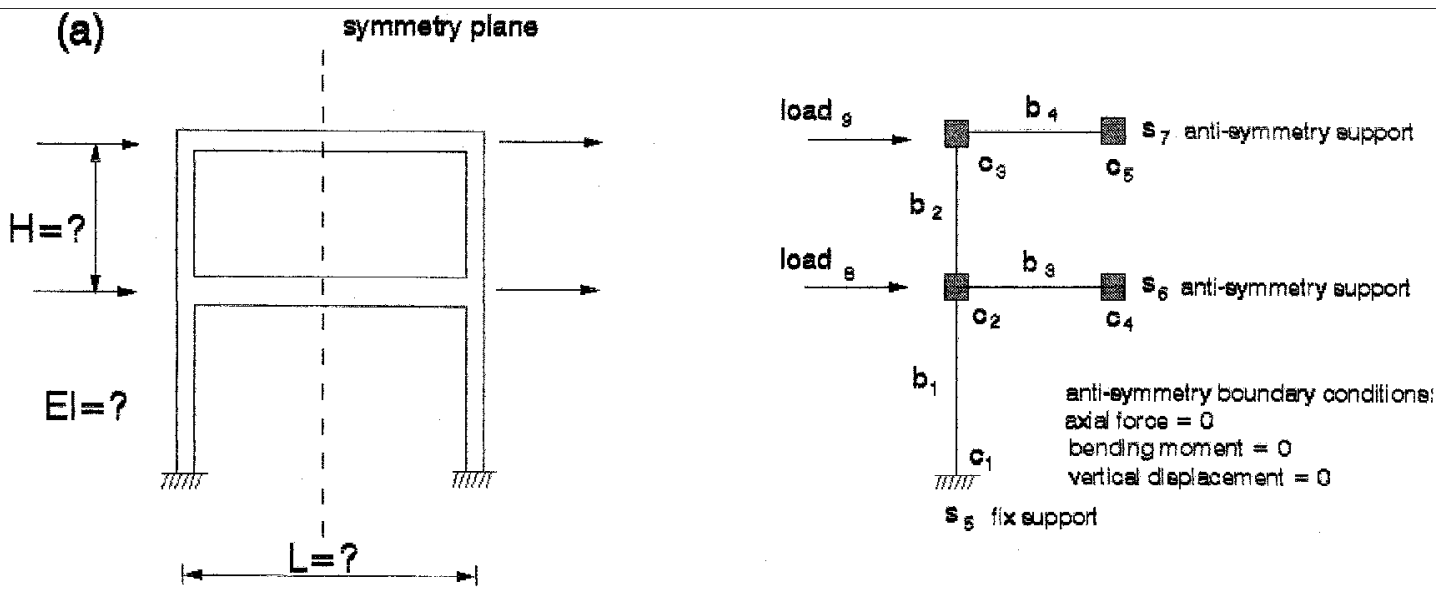

(b)

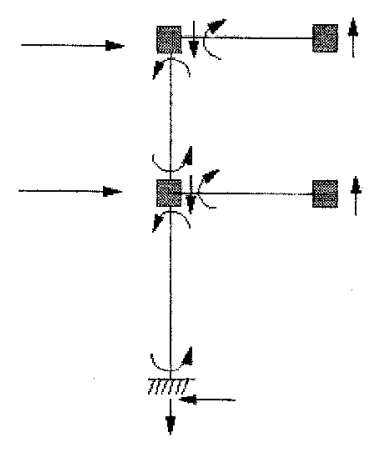

solution 1

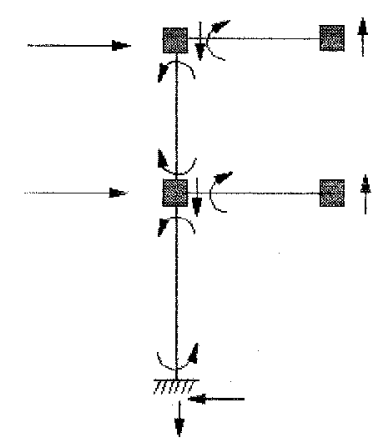

solution 2

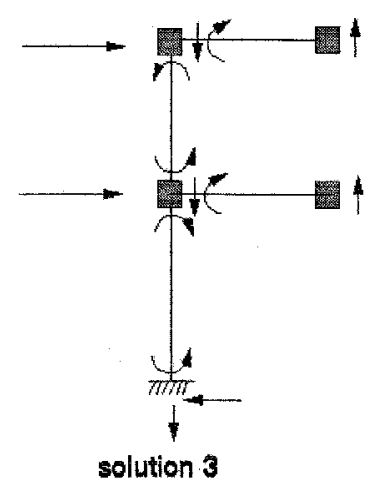

Figure 8. Two-story frame and the three solutions derived by Agrippa. 
difference in the three load transfer characteristics corresponds to single or double curvature in the columns. The first solution has both columns in double curvature. The second solution has the first-story columns in double curvature and the secondstory columns in single curvature. The third solution has the first-story columns in single curvature and the second-story columns in double curvature.

Even though there are three solutions, the behaviors share the following common features. One column is in compression, while the other is in tension, and the magnitude of the axial force is the same in both columns. The axial force in the first story is larger than the axial force in the second story and the shear force in the beams. The beams connecting the columns are subjected to shear, and the end moments follow a clockwise direction. The end moments at the bottom of the first-story columns follow a counterclockwise direction and are always larger than the end moments at the top of the first-story columns.

Presently, there is no indication in the program to know if the solutions correspond to initial section behavior characteristics or if they correspond to redistribution of forces due to material softening. By assuming that these solutions are caused by the initial section behavior characteristics, an engineering interpretation suggests that the first solution corresponds to a frame structure with similar section behavior characteristics. The third solution suggests the columns are shear walls with a section behavior corresponding to a much more rigid component than the beams. The second solution suggests a soft story because the section behavior for the second-story columns corresponds to a much more rigid component than the first-story columns. The second solution also suggests that the lateral loads at the second story are small in comparison with the lateral loads at the first story.

An engineering interpretation of the evaluation or heuristic rules may indicate that the likelihood of an axial force failure in the first-story columns is larger than the likelihood of an axial force failure for the second-story columns. It may also indicate that if the length of the beams is relatively short, there is a likelihood of shear failure caused by the bending moments at the connections with the columns. Finally, it should be pointed out that the example does not illustrate how the program can be used to derive relations between lengths or section behavior characteristics from desired load transfer characteristics.

The final application is symmetric three-dimensional frame resisting antisymmetric lateral loads, as illustrated in Figure $9 a$. The columns have the same length and section behavior characteristics about the $X, Y, Z$ axes. The beams along the $Y$ axis have the same length and section behavior characteristic, as do those along the $X$ axis. Figure $9 a$ also illustrates the model for the antisymmetric load that is used to evaluate the load transfer characteristics for the frame.

Agrippa derives one solution for the displaced shape and two solutions for the forces. The qualitative solution for joint displacements and corresponding displaced shape is presented in Figure 9b. Under the antisymmetric load, the displacements of the beams indicate that the frame rotates about the positive $z$ axis. The columns 
(a)

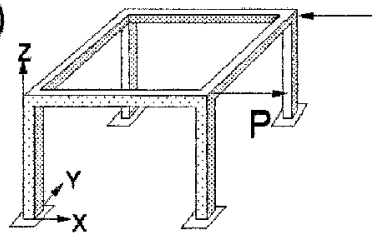

Frame structure

(b)

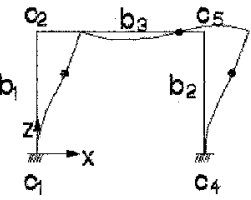

Front view

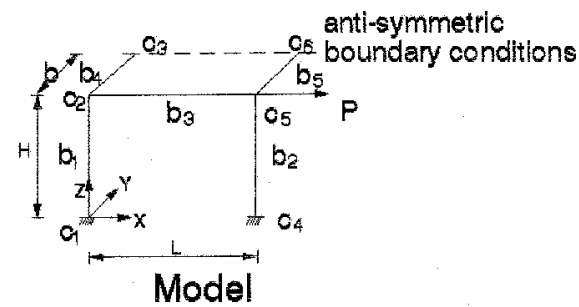

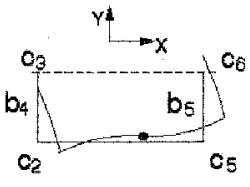

Top view

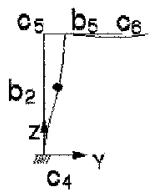

Side view

(c)
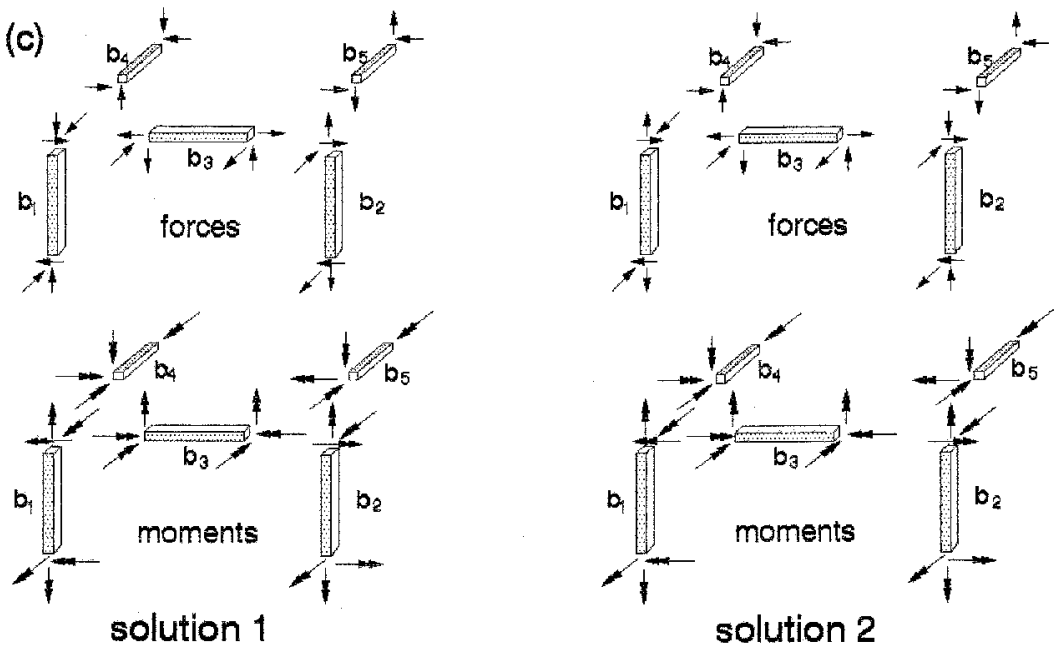

Figure 9. (a) Three-dimensional frame and corresponding model for an antisymmetric loading. (b) Displaced shape. (c) Load transfer characteristics. 
also have a torsional rotation along the positive $z$ axis. The connection $C_{5}$ has a positive $X$ displacement in the direction of the load.

There are two solutions for the direction of forces presented in Figure $9 c$. The two solutions are identical except for the axial forces in the columns. The first solution indicates that column $b_{1}$ is in compression and column $b_{2}$ is in tension; the second solution indicates that column $b_{1}$ is in tension and column $b_{2}$ is in compression. Both solutions have the following common features. The shear forces in the $X$ direction at the bottom of the columns oppose the load. The shear forces in the $Y$ direction have opposite signs, and the resultant moment caused by these two forces about the $Z$ axis opposes the moment due to the load. The torsional moments at the bottom of the columns also resist the torsional moment due to the external loads. The end moments about the $Y$ axis for the columns follow a negative direction, and the columns are in double curvature along the $Y$ axis. The end moments about the $X$ axis for column $b_{1}$ follow a negative direction, and the column is also in double curvature along the $X$ axis.

The beam $b_{3}$ is in tension and transfers shear forces as well as bending and torsional moments. The bending moments about the $Y$ axis are positive, and the beam is in double curvature along the $Y$ axis. The bending moments about the $Z$ axis are positive, and the beam is also in double curvature along the $Z$ axis. Beams $b_{4}$ and $b_{5}$ have no axial forces, but they transfer shear forces, bending moments, and torsional moments. The beams are in single curvature for the moments along the $X$ and $Z$ axes.

The axial forces in the columns are caused by the shear forces along the $Z$ axis in the beams. For example, the axial force in column $b_{1}$ depends on the relative magnitude of the moments from beams $b_{4}$ and $b_{3}$ about the $X$ and $Y$ axes, respectively, at connection $C_{2}$. The axial force in column $b_{1}$ also depends on the relative length of members $b_{4}, b_{3}$. A heuristic rule, "if the section behavior associated with a given parameter increases in stiffness, the parameter also increases its value," suggests the following engineering interpretation. If the section behavior along the $X$ axis for component $b_{4}$ increases in stiffness, the moment along the $X$ axis and shear force along the $Z$ axis at connection $C_{2}$ for component $b_{4}$ also increase. Consequently, the compression force in column $b_{1}$ increases. Similarly, if the section behavior along the $Y$ axis for beam $b_{3}$ increases in stiffness, the moment along the $Y$ axis and shear force along the $Z$ axis at connection $C_{2}$ for component $b_{3}$ also increase. Consequently, the tension force in column $b_{1}$ increases.

An order of magnitude reasoning suggests that if beam $b_{3}$ is rigid compared with beams $b_{4}$ and $b_{5}$, the load is transferred by the planar frame formed by components $b_{1}, b_{2}$, and $b_{3}$, and therefore column $b_{1}$ is in tension. In contrast, if beams $b_{4}$ and $b_{5}$ are rigid compared with beam $b_{3}$, the planar frame formed by components $b_{1}$ and $b_{4}$ transfers the load perpendicular to its plane. For this second extreme case the torsion in beams $b_{4}$ and $b_{5}$ increases. The bending along the $X$ axis for column $b_{1}$ and beam $b_{4}$ also increases, as does the compression force in column $b_{1}$. 


\section{CONCLUSIONS}

Qualitative reasoning is a methodology for evaluating the behavior of conceptual structural designs where limited information about the structure is available. The work presents a framework for qualitative structural analysis, which is implemented in the computer program Agrippa. This program derives load transfer characteristics and displacements for three-dimensional structures under static lateral and gravity loads. Reasoning with knowledge about topology and incomplete knowledge about geometry and material characteristics, Agrippa derives conclusions regarding the signs and relative magnitude of parameters such as internal forces, moments, displacements, and rotations. Future developments will include applications of the framework to tutoring structural analysis systems.

\section{REFERENCES}

Bozzo, L., and G. Fenves. 1992. Qualitative reasoning about structural behavior for conceptual design. Report No. UCB/SEMM-92/26, Structural Engineering Mechanics and Materials, University of California at Berkeley. Bredeweg, B., M. Reinders, and B. J. Wielinga. 1990. GARP: A unifying approach to qualitative reasoning: VF Memo 117, Department of Social Science Informatics, University of Amsterdam, The Netherlands.

Cohn, A. 1987. Qualitative reasoning. In Advanced topics in artificial intelligence, 2nd advanced course, $A C A I^{\prime} 87$ Oslo, Norway, July/August, ed. R. T. Norsum.

De Kleer, J., and J. Brown. 1984. A qualitative physics based on confluences. Artificial Intelligence 24:7-93.

Forbus, K. 1990. Qualitative physics: Past, present and future. In Readings in qualitative reasoning about physical systems, eds. Weld and J. De Kleer. San Mateo, Calif.: Morgan Kaufmann.

Oden, J. 1967. Linear and nonlinear analysis by conjugate analogy. Journal of the Structural Division, Proceedings of the American Society of Civil Engineers (August):27-45.

O'Keefe, R. 1990. The Craft of Prolog, Cambridge, Mass.: MIT Press. 